\section{Alternating flexed-extended posturing in progressive supranuclear palsy}

\author{
Nobuyuki Ishii, Hitoshi Mochizuki \\ Division of Neurology, Respirology, \\ Endocrinology and Metabolism, \\ Department of Internal Medicine, \\ University of Miyazaki, Japan
}

\begin{abstract}
A 69-year-old man who had been bedridden in nursing home because of a 5year history of progressive supranuclear palsy (PSP) was admitted due to aspiration pneumonia. Besides neck dystonia in extension, he showed "alternating flexed-extended posturing", in which the arm was flexed on one side and extended on the other Magnetic resonance imaging of the brain revealed global cerebral atrophy that predominantly affected the cortex and midbrain. The mechanisms of complex posturing in late-stage PSP may sometimes be related to decortication and decerebration as well as dystonia, and "alternating flexedextended posturing" might be one of the phenotypes of pathological progression in PSP.
\end{abstract}

\section{Introduction}

Patients with parkinsonism sometimes develop characteristic abnormal postures. Progressive supranuclear palsy (PSP), which is an atypical parkinsonian syndrome characterized by early postural instability, falls, vertical supranuclear palsy, and progressive dementia, usually causes diseasespecific postures such as neck dystonia in extension. ${ }^{1}$ Here, we report a patient with PSP who presented with "alternating flexed-extended posturing," in which the arm was flexed on one side and extended on the other. This posturing may potentially be caused by small differences in the degree of left $v s$. right neurodegeneration in the cerebral cortex and midbrain, both structures that are affected by PSP. ${ }^{1}$

\section{Case Report}

A 69-year-old man with a 5-year history of PSP was admitted due to aspiration pneumonia. When he was initially diagnosed with PSP, he exhibited rigidity of the left extremities and the trunk. As the disease progressed, he developed neck dorsiflexion followed by inversion of the left foot, suggesting dystonia. He had been bedridden in a nursing home for 2 years and had had difficulty communicating for 1 year.

On admission, he expressed no meaningful words and instead exhibited stereotyped moaning or groaning, which was caused by dysphonia and nonfluent aphasia. Neurological examination revealed bilateral vertical gaze palsy with preserved oculocephalic reflexes, axial rather than limb rigidity, preserved but sluggish bilateral pupillary light reflexes, and prominent neck dystonia. The deep tendon reflexes were exaggerated and the Babinski response was positive bilaterally. In addition, the upper extremities showed flexed posturing on the right side (Figure 1A, red arrowheads) and extended posturing on the left (red arrows), and the left lower limb exhibited flexion contracture. Magnetic resonance imaging of the brain revealed global cerebral atrophy that predominantly affected the cortex and midbrain (Figure 1B-D). The patient was treated with ampicillin/sulbactam for 7 days and discharged to a nursing home.

\section{Discussion}

PSP is the most common atypical parkinsonian syndrome, and typically presents with vertical supranuclear gaze palsy, postural instability with falls, and progressive cognitive disturbance. ${ }^{1}$ Patients with parkinsonism sometimes exhibit characteristic abnormal postures such as neck dystonia in extension in PSP, Pisa syndrome and camptocormia in Parkinson's disease, and dropped head in multiple system atrophy. 2,3 In addition to neck dystonia, our patient exhibited "alternating flexed-extended posturing," in which the arm was flexed on one side and extended on the other.

We speculate that this characteristic posturing may be caused by simultaneous decerebration and decortication as well as dystonia. Decerebrate posturing is caused by midbrain lesions that release the vestibulospinal postural reflexes from forebrain control and activate the extensor muscles, while decorticate posturing is due to dysfunction of the cerebral cortex that releases other spinal reflexes. ${ }^{4}$ These two postures are not disease-specific, and patients with neurodegenerative diseases usually manifest them in the bilateral limbs. Our patient, however, presented simultaneously with flexed posturing in the right limbs and extended posturing in the left limbs.

In contrast with the upper limbs, this patient's left hip and knee showed flexed posturing, and his foot was inverted. These postures were likely caused by dystonia,
Correspondence: Nobuyuki Ishii, Division of Neurology, Respirology, Endocrinology and Metabolism, Department of Internal Medicine, University of Miyazaki, 5200 Kihara, Kiyotake, Miyazaki, 889-1692, Japan. Tel: +81-985-85-2965 - Fax: +81-985-85-1869 E-mail: nobuyuki_ishii@med.miyazaki-u.ac.jp

Key words: progressive supranuclear palsy; dystonia; decerebrate state; decorticate state; abnormal posturing.

Contributions: NI and HM, design and coordination of the study, analysis and interpretation of the data, collection of the material, drafting of the manuscript.

Conflict of interest: The authors declare no potential conflict of interest.

Funding: This study was partly supported by JSPS KAKENHI Grant Number 19K20715 (NI).

Received for publication: 27 July 2019.

Accepted for publication: 12 August 2019.

This work is licensed under a Creative Commons Attribution NonCommercial 4.0 License (CC BY-NC 4.0).

(C) Copyright: the Author(s), 2019

Licensee PAGEPress, Italy

Neurology International 2019; 11:8257

doi:10.4081/ni.2019.8257

which often accompanies PSP, 5 rather than by decortication or decerebration. In the early disease stage, the patient exhibited rigidity in the left limbs, which was predictive of left limb dystonia and fixed contracture of the left lower limbs. The right upper limb exhibited flexed posturing, which could not differentiate decorticate and dystonic posturing. It is impossible to exclude the possibility that dystonia may have contributed to the final posturing.

The laterality of the patient's abnormal posturing is likely related to the pathological progression of PSP. PSP causes degeneration of both the cerebral cortex and midbrain, which leads, for example, to dementia and supranuclear gaze palsy, respectively. ${ }^{1}$ In our patient, besides dystonia, slight differences in pathological progression in the cortex and midbrain may have caused concurrent decorticate and decerebrate posturing in the late clinical course. Furthermore, the prominent asymmetrical posture might indicate a possible diagnosis of PSP with corticobasal syndrome (PSPCBS) rather than classic PSP (Richardson syndrome). ${ }^{6}$ 


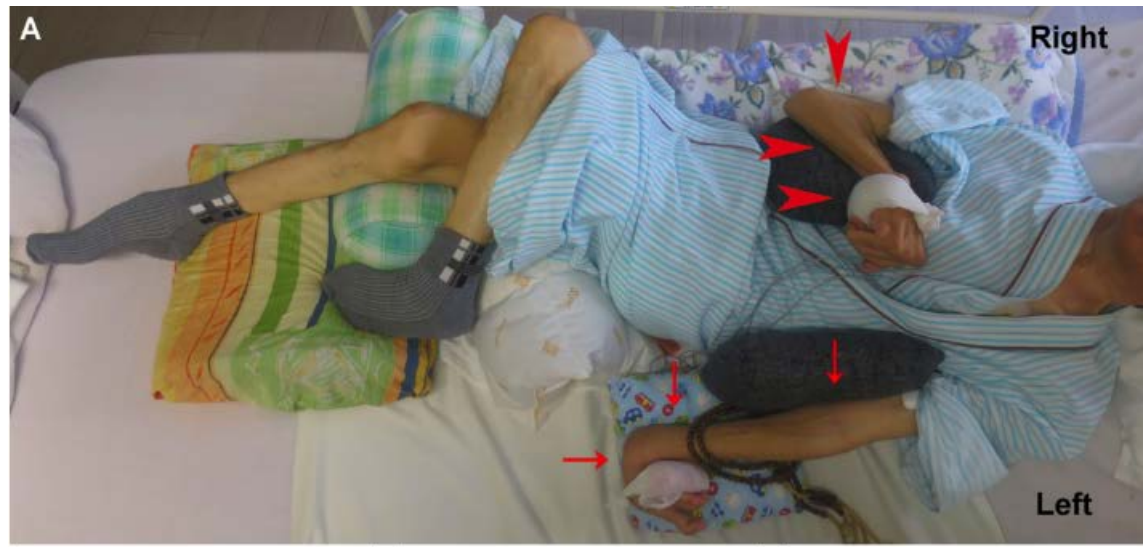

\section{References}

1. Williams DR, Lees AJ. Progressive supranuclear palsy: clinicopathological concepts and diagnostic challenges. Lancet Neurol 2009;8:270-9.

2. Savica R, Kumar N, Ahlskog JE, et al. Parkinsonism and dropped head: dystonia, myopathy or both? Parkinsonism Relat Disord 2012;18:30-4.

3. Doherty KM, van de Warrenburg BP, Peralta MC, et al. Postural deformities in Parkinson's disease. Lancet Neurol 2011;10:538-49.
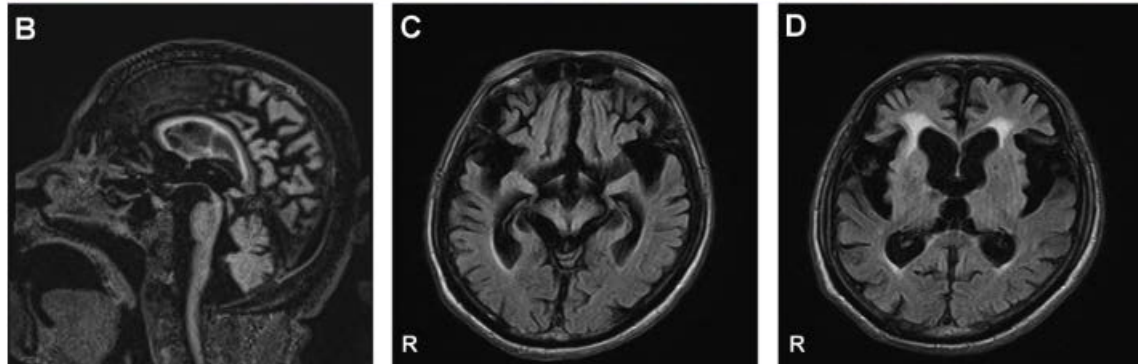

4. Posner JB, Plum F. Plum and Posner's diagnosis of stupor and coma. fourth ed. Oxford University Press, New York, 2007.

5. Barclay CL, Lang AE. Dystonia in progressive supranuclear palsy. J Neurol Neurosurg Psychiatry 1997;62:352-6.

6. Ling H, de Silva R, Massey LA, et al. Characteristics of progressive supranuclear palsy presenting with corticobasal syndrome: a cortical variant. Neuropathol Appl Neurobiol 2014;40:149-63.

Figure 1. A) Alternating flexed-extended posture in the upper limbs; flexed right arn (arrowheads) and extended left arm (arrows). B-D) Fluid-attenuated inversion recovery (FLAIR) MRI with diffuse atrophy in the midbrain and cerebral cortex.

\section{Conclusions}

The mechanisms of complex posturing in late-stage PSP may sometimes be related to decortication and decerebration as well as dystonia, and "alternating flexed-extended posturing" might be one of the phenotypes of pathological progression in PSP, especially PSP-CBS. 